[8] K. M. Evenson, D. A. Jennings, F. R. Petersen, and J. O. Henningsen, in preparation. Thanks are due to the Laser Physics Section of NBS for its hospitality.

[9] Y. Y. Kwan and D. M. Dennison, "Analysis of the torsion-rotation spectra of the isotopic methanol molecules," J. Mol. Spectrosc., vol. 43, pp. 291-319, Aug. 1972.

[10] A. Serrallach, R. Meyer, and Hs. H. Günthard, "Methanol and deuterated species: Infrared data, valence force field, rotamers and conformation," J. Mol. Spectrosc., vol. 52, pp. 94-129, 1974.
[11] D. G. Burkhard and D. M. Dennison, "Rotation spectrum of methyl alcohol," J. Mol. Spectrosc. vol. 3, pp. 299-334, Aug. 1959.

[12] R. G. Lee, R. H. Hunt, E. H. Plyler, and D. M. Dennison, "A high resolution study of the OH stretch fundamental of methanol," J. Mol. Spectrosc., vol. 57, pp. 138-154, July 1975.

[13] C. O. Weiss, M. Grinda, and K. Siemsen, "FIR laser lines of $\mathrm{CH}_{3} \mathrm{OH}$ pumped by $\mathrm{CO}_{2}$ laser sequence lines," IEEE J. Quantrum Electron., vol. QE-13, p. 892, Nov. 1977.

\title{
Optical Parametric Oscillator Dependence on Pump Laser Beam Quality
}

\author{
THEODORE F. EWANIZKY
}

\begin{abstract}
Experimentation with a pulsed, angle-tuned $\mathrm{LiNbO}_{3}$ optical parametric oscillator revealed the dependence of pump power depletion and conversion efficiency on pump laser beam divergence. Mathematical expressions, developed for these parameters, were based on a spherical-wave model. Calculated results and comparison with experimental observations indicate the degree to which phasefront curvature affects parametric oscillator performance.
\end{abstract}

\section{INTRODUCTION}

$\mathrm{E}$ XPERIMENTATION and analysis were conducted in an effort to determine the effects of multimode pumping on the operating characteristics of a doubly resonant optical parametric oscillator (OPO). This question naturally arises if an OPO is considered for application as an auxiliary system, to provide wavelength diversity for a primary laser which, however, may have less than ideal characteristics as a pump source.

The apparently anomolous values of OPO conversion efficiency and pump power depletion which were measured, could not be satisfactorily explained in terms of plane-wave analyses. Experimentation revealed an expected functional dependence of OPO performance on pump laser beam quality. A simplified theoretical model was adopted, and appropriate mathematical expressions were derived, which illustrate how phasefront curvature produces substantial increase of OPO threshold, and resulting deviation in the operating parameters.

\section{Experimental Procedure}

Initial experimentation consisted of measuring the output power of an angle tuned, doubly resonant $\mathrm{OPO}$ using a $5 \mathrm{~cm}$

Manuscript received February 2, 1978; revised June 2, 1978.

The author is with the U.S. Army Electronics Research and Development Command, Fort Monmouth, NJ 07703. long lithium niobate crystal (similar to that described by Herbst et al. [1]), pumped by the raw beam, multimode output of a $Q$-switched Nd:YAG laser. The pump laser beam was approximately $0.6 \mathrm{~cm}$ in diameter, with a near-field intensity distribution that appeared to be highly multimode. Despite amplitude fluctuations, intensity falloff was sharp at the edge of the beam, and would be more closely approximated by uniform illumination than a Gaussian function. The full angle far-field angular divergence was measured to be $4 \mathrm{mrad}$. No effort was made to restrict the spectral width of the $1.06 \mu \mathrm{m}$ pump radiation. The OPO resonance cavity consisted of closely spaced plane mirrors, $7 \mathrm{~cm}$ apart, with high reflectivity at $2.13 \mu \mathrm{m}$ and $>90$ percent transmission at the $1.065 \mu \mathrm{m}$ pump wavelength. Mirror reflectivity decreased symmetrically on either side of $2.13 \mu \mathrm{m}$, thus limiting the spectral tuning range to some degree. Close mirror spacing was found to be a crucial factor because of the short pump pulse duration. With the space limitation thus imposed, temperature control equipment could not be utilized and the OPO crystal was simply allowed to assume ambient temperature. Energy conversion efficiencies as high as 16 percent could be attained near degeneracy, for pump pulses of $15 \mathrm{~ns}$ full width at half maximum (FWHM) duration, with $120 \mathrm{~mJ}$ energy, operating at 10 pulses per second (pps).

Near degeneracy, broad OPO spectral bandwidth with a double cluster was observed, indicating doubly resonant operation. For example, two broad signal peaks observed at 2.055 and $2.104 \mu \mathrm{m}$ were partly coalesced and extended over a total bandwidth of $265 \mathrm{~cm}^{-1}$. Signal bandwidth remained relatively broad but decreased toward shorter wavelengths and at $1.866 \mu \mathrm{m}$, where the bulk of experimentation was conducted, a single peak at $54 \mathrm{~cm}^{-1}$ wide was typically observed. The shift from cluster to single peak operation may be explained 


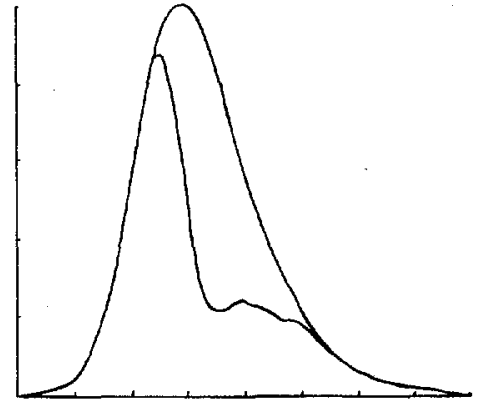

(a)

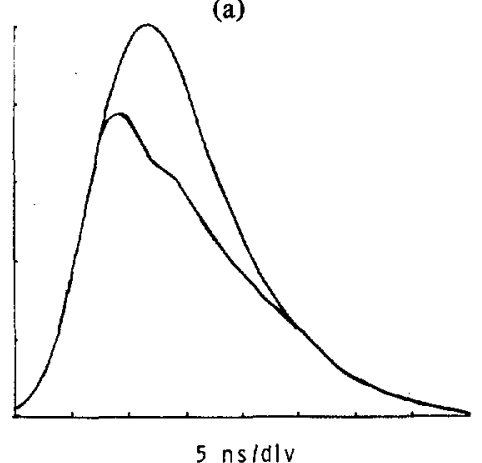

(b)

Fig. 1. Superimposed incident and transmitted pump pulses. (a) Total transmitted power; (b) Spatially filtered, 1 mrad maximum angular divergence.

[2] by the increased OPO threshold encountered at wavelengths away from degeneracy. In addition to the gain detuning factor, diminishing mirror reflectivity also produced a progressively increasing threshold. Generally broad spectral width was apparently due to the combined effects of pump laser beam divergence and multilongitudinal mode frequency content. Since the cavity mirrors presented substantial reflectivity at both the signal and idler wavelengths over the experimental tuning range, and no specific measures were taken to inhibit recombination generation of pump radiation, it was assumed that the OPO was operating in the doubly resonant mode, with power dependent reflections.

The total transmitted pump radiation was observed with a fast-rise detector and oscilloscope. Fig. 1(a) shows typical incident and transmitted pump pulses, superimposed for comparison. A quasi-steady-state transmitted power level can be seen in the depleted pulse, for a short period near the peak incident power. Note that a relatively small degree of pump depletion is exhibited, despite the high incident power produced by the $Q$-switched pump laser. Direct deduction of OPO threshold and conversion efficiency from the depleted power level leads to apparently anomolous values.

Next, the transmitted beam was passed through a well corrected, long focal length lens and its simulated far-field power distribution studied at the lens focal plane. Without OPO operation, burns made on Polaroid print film were circularly symmetric, with roughly Gaussian profile, and a spot size compatible with the measured pump beam angular divergence. With the crystal oriented to produce efficient OPO generation, however, decreased burn intensity within the central region of the pattern could be clearly discerned. This observation qualitatively demonstrated OPO dependence on pump laser beam quality.

To obtain a more quantitative measure, various diameter circular apertures were aligned with the beam at the focal plane. By this means, radiation was sampled in the simulated far field, according to its near-field propagation direction [3] . For a lens of focal length $f$, radiation propagating within a cone of internal angle $\phi$ will produce a spot of diameter $d=f \phi$ at the focal plane. Fig. 1(b) illustrates the depleted pulse observed for such spatially filtered radiation, with $1 \mathrm{mrad}$ full-angle divergence. Despite the fractional power contained within the $1 \mathrm{mrad}$ component, substantially greater pump depletion was observed, presumably due to the more efficient interaction of the low divergence radiation. Measurements made with various aperture sizes confirmed this general trend. Thus, the apparent OPO threshold power, signified by the quasi-steady-state depletion, was seen to have a functional dependence on pump laser beam divergence. For this reason, quantitative estimates of OPO threshold power or conversion efficiency using previous analyses [4], [5] would be inappropriate since they are based on the interaction of waves with planar phasefronts.

\section{Theoretical Calculations}

Zernike and Midwinter [6] suggest an analytical approach for estimating second-harmonic generation efficiency when only the pump laser beam divergence characteristics are known and not the exact transverse phase distribution. In a similar vein, appropriate mathematical expressions for pump depletion and OPO conversion efficiency were developed that would reflect the effects of phasefront curvature and correspond, at least qualitatively, to the experimental observations.

A circularly truncated portion of a uniform intensity spherical wave was adopted as the theoretical model to represent the actual pump beam. To achieve physical correlation, the truncated wave is considered to have an equal cross-sectional radius $a$ and a radius of curvature $R=2 a / \phi$, where $\phi$ is the angular divergence of the pump radiation component measured in each experimental case.

Assume the phasefront normal at the center of the wave to be properly oriented for perfect index-matching at some angle $\theta_{m}$ with respect to the principal axis of the OPO crystal. Then, at other locations on the spherical surface it would have some angular misorientation $\Delta \theta$. The effect of this misorientation is to encounter a variation in refractive index for the $e$-polarized pump radiation, and hence produce an index mismatch given by

$$
\Delta k=2 \pi\left[\frac{n_{p}^{e}\left(\theta_{m}+\Delta \theta\right)}{\lambda_{p}}-\frac{n_{s}^{0}}{\lambda_{s}}-\frac{n_{i}^{0}}{\lambda_{i}}\right]
$$

$\left(n_{s}^{0}\right.$ and $n_{i}^{0}$ are the indices for the 0 -polarized signal and idler waves). Since $\mathrm{LiNbO}_{3}$ is a uniaxially birefringent medium, the above effect occurs only for $k$-vector misorientation in $\theta$. Azimuthal misorientation, in a direction normal to the plane containing $\theta_{m}$, produces a relatively negligible degree of mis- 
match and may be ignored for small $\Delta \theta$. For colinear indexmatching, at fixed wavelengths $\lambda_{p}, \lambda_{s}$ and $\lambda_{i}$, small-signal OPO gain is proportional to the factor

$$
G(\Delta k)=\operatorname{sinc}^{2}(\Delta k L / 2)
$$

where $L$ is the crystal length. Therefore, OPO threshold variation due to index-mismatch can be readily expressed in terms of the plane-wave threshold intensity $I_{0}$ by the relation

$$
I_{t}(\Delta k)=I_{0} / G(\Delta k) \text {. }
$$

The pump beam is generally not at normal incidence to the surface of the OPO crystal, and the crystalline axis is a nominal angle $\alpha=43^{\circ}$ to the surface. Consequently, the refracted angle $\rho$ must be calculated for $k$-vector incident angle $i$. It was found that refraction has an important effect, since it tends to reduce $\Delta \theta$ within the crystalline medium. In a similar derivation to that in [7], it can be shown that the $k$-vector refraction angle may be solved by quadratic formula from the expression

$$
\begin{aligned}
& \left(0^{2} \sin ^{2} \alpha+\epsilon^{2} \cos ^{2} \alpha\right) \sin ^{2} i \cdot \cot ^{2} \rho \\
& -\left(\epsilon^{2}-0^{2}\right) \sin 2 \alpha \cdot \sin ^{2} i \cdot \cot \rho \\
& \quad+\left(0^{2} \cos ^{2} \alpha+\epsilon^{2} \sin ^{2} \alpha\right) \sin ^{2} i=1,
\end{aligned}
$$

where

$$
0=1 / n_{0}, \epsilon=1 / n_{\epsilon} \text {. }
$$

For a doubly resonant OPO (with power-dependent reflection), the relationship between the incident and transmitted pump intensities $I$ and $I^{\prime}$, respectively, depends on the conditions

$$
\begin{aligned}
& I^{\prime}=I_{t}, \text { for } I>I_{t} \\
& I^{\prime}=I, \text { for } I<I_{t} .
\end{aligned}
$$

Appropriately modifying the generalized expressions for plane-wave interactions given by Bjorkholm [5], in view of the above considerations, new equations for the pump depletion and conversion efficiency can be derived.

If $r=\Delta \theta \cdot R$ is defined as the radial dimension on the phasefront surface, in a direction parallel to the plane containing the crystalline axis, the relation for the normalized pump depletion becomes

$$
\begin{aligned}
\frac{P^{\prime}}{P}= & 1+\frac{2}{\pi a^{2}}\left[\left(\frac{P_{t}}{p}\right) \int_{-a}^{a} \frac{\left(a^{2}-r^{2}\right)^{1 / 2}}{G(r)} d r\right. \\
& \left.-\int_{-a}^{a}\left(a^{2}-r^{2}\right)^{1 / 2} d r\right] .
\end{aligned}
$$

The ratio of transmitted to incident pump power $P^{\prime} / P$ is obtained by integration over the phasefront surface. It is seen to be influenced by the ratio of incident to threshold power $P / P_{t}$ and the phasefront curvature through the relative gain function $G(r)$. Numerical integration sums over the incremental areas with respect to the test condition

$$
G(r) \geqslant P_{t} / P \text {. }
$$

Contributions to both integrals are accumulated only if (8) is satisfied at some value of $r$. The test condition just evaluates whether the incident power exceeds the effective threshold produced by $G(r)$, and follows from (3). The conversion efficiency is defined as the ratio of combined signal and idler intensities to that of the incident pump wave. For the same considerations formerly applied, the corresponding expression becomes

$$
\begin{aligned}
\eta= & \frac{4}{\pi a^{2}}\left(\frac{P_{t}}{P}\right)^{1 / 2}\left[\int_{-a}^{a}\left(\frac{a^{2}-r^{2}}{G(r)}\right)^{1 / 2} d r\right. \\
& \left.-\left(\frac{P_{t}}{P}\right)^{1 / 2} \int_{-a}^{a}\left(\frac{a^{2}-r^{2}}{G(r)}\right) d r\right] .
\end{aligned}
$$

The calculational procedure in this case is also that contributions are made to both integrals only if (8) is satisfied. This just means that parametric conversion occurs only in spatial regions where the incident pump intensity is over threshold.

Fig. 2 shows the calculated results for $P^{\prime} \mid P$ and $\eta$ for extended values of $P / P_{t}$. Each case shown models experimental observations for radiation with full-angle divergence of 1,2 , and $4 \mathrm{mrad}$ (the latter value corresponding to the unfiltered pump beam). Curves representing plane-wave interaction are included for comparison. The inflections visible on some of the curves arise at sufficiently high values of $P / P_{t}$ for which regions of the phasefront, at greater curvature, exceed threshold at the secondary maxima of the $G(\Delta k)$ function. This implies corresponding transverse amplitude variations in both the transmitted pump wave and the parametrically generated radiation.

The mathematical expression of the pump depletion in the case of a singly resonant OPO may be derived in a similar manner. For plane-wave excitation, the transmitted pump intensity is given by [5]

$$
I^{\prime}=I \cos ^{2} \Gamma \text {, for } I>I_{t},
$$

subject to

$$
\frac{\sin ^{2} \Gamma}{\Gamma^{2}}=\frac{I_{t}}{I}
$$

In view of (3), (11) becomes

$$
\sin \Gamma=\left[\frac{1}{G(r)}\left(\frac{P_{t}}{P}\right)\right]^{1 / 2} \Gamma .
$$

Then, the expression for the pump depletion of a singly resonant OPO becomes

$$
\begin{aligned}
\frac{P^{\prime}}{P}= & 1+\frac{2}{\pi a^{2}}\left[\int_{-a}^{a} \cos ^{2} \Gamma \cdot\left(a^{2}-r^{2}\right)^{1 / 2} d r\right. \\
& \left.-\int_{-a}^{a}\left(a^{2}-r^{2}\right)^{1 / 2} d r\right] .
\end{aligned}
$$

Using numerical integration, contributions to the integrals are accumulated only if the test condition (8) is satisfied. 


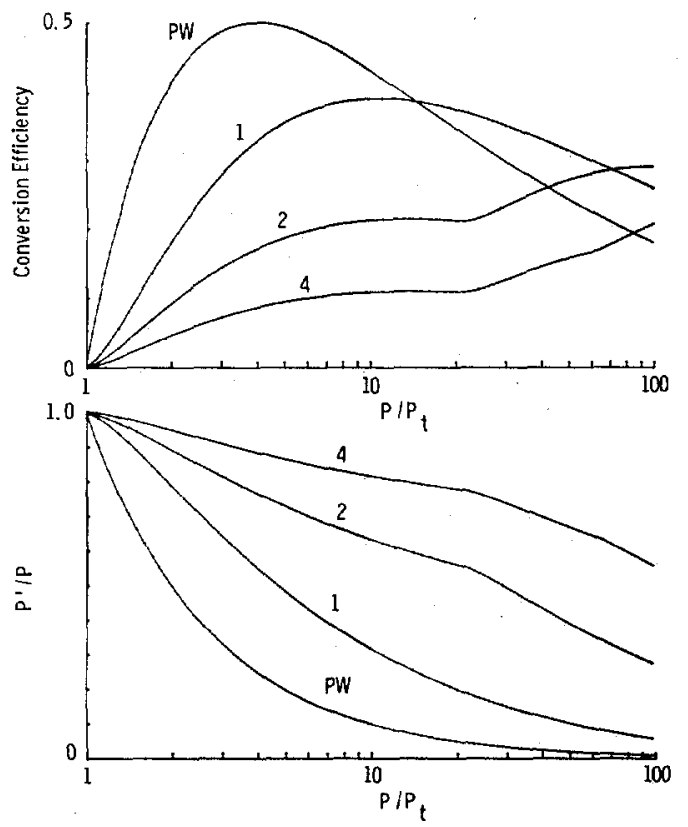

Fig. 2. Doubly resonant OPO pump depletion and parametric conversion efficiency for spherical pump waves with $0.5,1$, and $2 \mathrm{mrad}$ maximum $k$-vector misorientation (corresponding to 1,2 , and 4 mrad full-angle divergence). Plane-wave curves are shown for comparison.

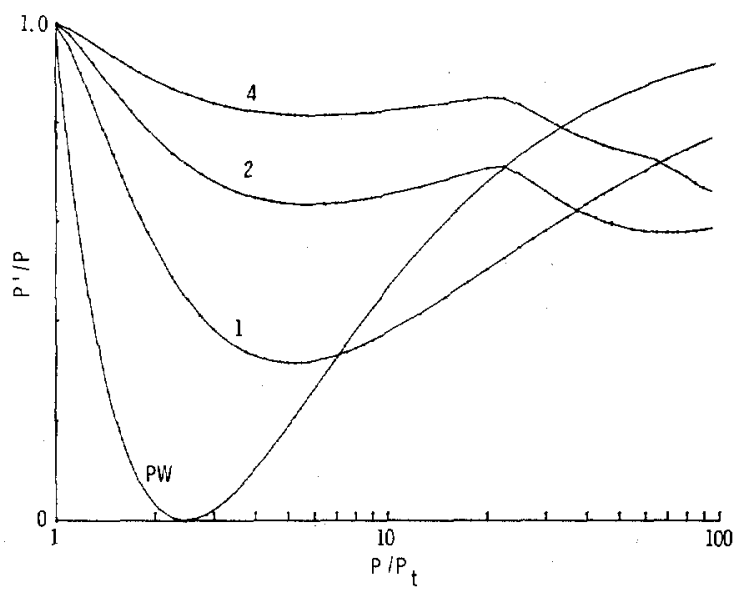

Fig. 3. Singly resonant OPO pump depletion.

The calculated results are shown in Fig. 3 for the same cases in Fig. 2. The effects of phasefront sphericity are clearly evident. Compared to the plane-wave case, maximum conversion efficiency is diminished and shifted to higher values of $P / P_{t}$.

\section{Discussion AND CONCLUSIONS}

The calculations are based on a model which considers interaction of a fixed, discrete set of wavelengths. In actuality, the real pump beam was not spectrally restricted and consisted of multilongitudinal-mode radiation. However, this discrepancy may be ameliorated to some degree by mode competition within the short duration of the pump pulse [5] , [8] .

Despite inadequacies of the theoretical model, it is interesting to make some quantitative comparison with the experimental data. From Fig. 1(b), the pump depletion, measured slightly beyond peak power, is about 0.27 . The curve of Fig. 2, representing a maximum $1 \mathrm{mrad}$ divergence, gives $P / P_{t}=13$ for this value. Separate beam divergence measurements showed that 35 percent of the total beam power was contained within a cone of $1 \mathrm{mrad}$ internal angle. Combining these values gives an incident pump threshold intensity of $7.6 \times 10^{5} \mathrm{~W} / \mathrm{cm}^{2}$. At the same value of $P / P_{t}$, the calculated conversion efficiency is 39 percent. In contrast, the maximum depletion for the raw beam, measured near peak power, is 0.67 . This gives $P / P_{t}=$ 49 , with a calculated conversion efficiency of 15.8 percent.

It is interesting to note that all of the above figures are reasonable in their proportions. An additional feature of the calculations suggests an explanation for the experimental observation that saturation of the conversion efficiency could not be induced. That is, OPO output monotonically increased with pump laser power, up to its maximum capability. The curves indicate that because of phasefront curvature, regeneration of pump radiation (power-dependent reflection) is also hampered, and peak efficiency is attained at relatively large $P / P_{t}$ compared with plane-wave interactions.

Taken as a guide, the results graphically illustrate the consequences of pump laser beam divergence, or phasefront curvature, on OPO conversion efficiency. They also indicate the degree to which this parameter can cause apparent discrepancy if pump power depletion is used as an analytic tool for quantitative measurements.

In view of these considerations, for a pulsed OPO with unfocused pump radiation, an ideal choice for the pump laser design might incorporate an unstable resonator optical configuration. It has been shown [9] that near diffraction-limited beam quality can be directly obtained from an oscillator stage, without sacrifice of overall operating efficiency. Most recently, in fact, Herbst et al. [10] have found that the annular nearfield beam from an unstable resonator laser was more efficient for OPO excitation than an equivalent energy Gaussian beam.

\section{ACKNOWLEDGMENT}

The author gratefully acknowledges valuable discussions with Drs. G. R. Osche and R. L. Herbst, the encouragement of Dr. R. G. Buser, and able technical assistance of Mr. W. Saltzman and Mrs. C. Amato.

\section{REFERENCES}

[1] R. L. Herbst, R. N. Fleming, and R. L. Byer, "A 1.4-4 $\mu \mathrm{m}$ highenergy angle-tuned $\mathrm{LiNbO}_{3}$ parametric oscillator," Appl. Phys. Lett., vol. 25, pp. 520-522, Nov. 1974; also see R. L. Byer and R. L. Herbst, "Parametric oscillation and mixing," Topics in Applied Physics, vol. 16. New York: Springer, 1977, pp. 81-137.

[2] J. E. Bjorkholm, "Some spectral properties of doubly and singly resonant pulsed optical parametric oscillators," Appl. Phys. Lett., vol. 13, pp. 399-401, Dec. 15, 1968.

[3] M. Borne and E. Wolf, Principles of Optics. London: Pergamon, 1970, p. 384.

[4] G. D. Boyd and D. A. Kleinman, "Parametric interaction of focused Gaussian light beams," J. Appl. Phys., vol. 39, pp. 35973639 , July 1968.

[5] J. E. Bjorkholm, "Some effects of spatially nonuniform pumping in pulsed optical parametric oscillators," IEEE J. Quantum Electron., vol. QE-7, pp. 109-118, Mar. 1971. 
[6] F. Zernike and J. E. Midwinter, Applied Non-Linear Optics. New York: Wiley, 1973, ch. 5 .

[7] - Applied Non-Linear Optics. New York: Wiley, 1973, ch. 1.

[8] J. E. Bjorkholm and H. G. Danielmeyer, "Frequency control of a pulsed optical parametric oscillator by radiation injection," Appl. Phys. Lett., vol. 15, pp. 171-173, Sept. 1969.
[9] T. F. Ewanizky and J. M. Craig, "Negative-branch unstable resonator Nd:YAG laser," Appl. Opt., vol. 15, pp. 1465-1469, June 1976.

[10] R. L. Herbst, H. Komine, and R. L. Byer, "A $200 \mathrm{~mJ}$ unstable resonator Nd:YAG oscillator," Opt. Commun., vol. 21, pp. 5-7, Apr. 1977.

\title{
Resonantly Enhanced $26 \mathrm{GHz}$ Parametric Upconversion of $\mathrm{CO}_{2}$ Laser in $\mathrm{NH}_{3}$
}

\author{
CHARLES K. ASAWA AND RICHARD L. ABRAMS, MEM BER, IEEE
}

\begin{abstract}
Resonantly enhanced three-wave mixing of the $10.784 \mu \mathrm{m}$ $R(18)$ line of the $\mathrm{C}^{13} \mathrm{O}_{2}^{16}$ laser and microwave radiation at $26.378 \mathrm{GHz}$ in Stark tunable $\mathrm{N}^{14} \mathrm{H}_{3}$ has resulted in upconverted parametric output at $10.774 \mu \mathrm{m}$. The energy levels of $\mathrm{N}^{14} \mathrm{H}_{3}$ involved in the interaction and the apparatus used in the experiment are described. Experimental results showing saturation of the parametric signal with increasing $\mathrm{N}^{14} \mathrm{H}_{3}$ pressure, input microwave power, and input laser power are described.
\end{abstract}

\section{INTRODUCTION}

$\mathrm{T}$ HREE-WAVE mixing in gaseous media has been shown to be possible in the presence of a dc electric field [1]. Under conditions of resonance enhancement, three-wave mixing in Stark tunable gases was predicted [1] and observed [2] in $\mathrm{NH}_{2} \mathrm{D}$, where the dc electric field served to break inversion symmetry as well as to tune the energy levels into resonance with the applied frequencies. Single-sideband downconversion by $4.02 \mathrm{GHz}$ of a $10.59 \mu \mathrm{m} \mathrm{CO}$ laser was observed with conversion efficiencies as high as 0.2 percent. The dependence of the signal on dc field, $\mathrm{NH}_{2} \mathrm{D}$ pressure, and microwave power were reported.

We report here measurements of three-wave mixing in a new molecular system which offers the potential for increased conversion efficiency as well as operation at much higher microwave frequency. The $R(18)$ line at $10.784 \mu \mathrm{m}$ of the $\mathrm{C}^{13} \mathrm{O}_{2}^{16}$ laser and $26.4 \mathrm{GHz}$ microwave radiation are mixed in ordinary ammonia gas, $\mathrm{N}^{14} \mathrm{H}_{3}$, resulting in the parametric generation of radiation at a new frequency, $10.774 \mu \mathrm{m}$. The mixing is resonantly enhanced by applying an appropriate Stark electric

\footnotetext{
Manuscript received June 12,1978. This research was supported in part by DARPA and monitored by the Office of Naval Research.

The authors are with the Hughes Research Laboratories, Malibu, CA 90265.
}

field to bring the energy levels of $\mathrm{N}^{14} \mathrm{H}_{3}$ into resonance with all three waves. The $10.784 \mu \mathrm{m}$ resonance transition in ammonia exhibits an unusually large absorption coefficient [3] and thus a large nonlinear mixing coefficient; the large value permitted observation of new features during the experimental study, including laser power saturation, microwave power saturation as well as pressure dependence of the parametric interaction.

\section{Ammonia Gas Resonance Transitions}

The three energy levels and transitions of $\mathrm{N}^{14} \mathrm{H}_{3}$ involved in the resonance parametric interaction described here are shown in Fig. 1 along with the corresponding Stark absorption spectrum. The lowest two levels are the symmetric $(s)$ and the asymmetric $(a)$ inversion pair states, lying approximately 288 $\mathrm{cm}^{-1}$ above the lowest ground state, assigned as $\left(v_{2}=0, J=6\right.$, $K=6, s$, and $a$ ). The third level is an excited symmetric state assigned as $\left(v_{2}=1, J=6, K=6, s\right)$, the assignment being based upon the $\mathrm{C}^{13} \mathrm{O}_{2}^{16}$ laser line tabulation by Freed et al. [4] and the spectroscopic study of $\mathrm{N}^{14} \mathrm{H}_{3}$ by Shimizu [5]. The $\left(v_{2}=\right.$ $0, J=6, K=6, s$, and $a$ ) inversion pair will be called the "ground" states in the discussion here.

The ground inversion pair states are mixed and split into seven upper and seven lower components with the application of a Stark electric field. The components are designated by $|M|=6,5, \cdots, 0$, where each component is a mixture of the symmetric and asymmetric pair states due to the electric field perturbation. The excited upper $\left(v_{2}=1, J=6, K=6, s\right)$ symmetric state is not split by the Stark field within our resolution due to the large zero field inversion splitting of the excited state.

On applying a Stark field of $\sim 6560 \mathrm{~V} \cdot \mathrm{cm}^{-1}$, the upper ground $|M|=6$ level and the excited $|M|=6$ level are brought into resonance with the $10.784 \mu \mathrm{m} R(18)$ line of the $\mathrm{C}^{13} \mathrm{O}_{2}^{16}$ 